Math. Model. Nat. Phenom.

Vol. 7, No. 3, 2012, pp. 99-116

DOI: $10.1051 / \mathrm{mmnp} / 20127308$

\title{
Deterministic Chaos vs. Stochastic Fluctuation in an Eco-epidemic Model
}

\author{
P.S. Mandal ${ }^{1}$, M. Banerjee ${ }^{2 *}$ \\ ${ }^{1,2}$ Department of Mathematics and Statistics \\ Indian Institute of Technology, Kanpur \\ Kanpur - 208016, INDIA
}

\begin{abstract}
An eco-epidemiological model of susceptible Tilapia fish, infected Tilapia fish and Pelicans is investigated by several author based upon the work initiated by Chattopadhyay and Bairagi (Ecol. Model., 136, 103 - 112, 2001). In this paper, we investigate the dynamics of the same model by considering different parameters involved with the model as bifurcation parameters in details. Considering the intrinsic growth rate of susceptible Tilapia fish as bifurcation parameter, we demonstrate the period doubling route to chaos. Next we consider the force of infection as bifurcation parameter and demonstrate the occurrence of two successive Hopfbifurcations. We identify the existence of backward Hopf-bifurcation when the death rate of predators is considered as bifurcation parameter. Finally we construct a stochastic differential equation model corresponding to the deterministic model to understand the role of demographic stochasticity. Exhaustive numerical simulation of the stochastic model reveals the large amplitude fluctuation in the population of fish and Pelicans for certain parameter values. Extinction scenario for Pelicans is also captured from the stochastic model.
\end{abstract}

Keywords and phrases: Eco-epidemiology, stability, Hopf-bifurcation, chaos, stochasticity, extinction

Mathematics Subject Classification: 34C23, 60J25, 92D25

\section{Introduction}

Among various types of interacting population models, prey-predator type interaction models have received significant attention in the area of mathematical ecology after the pioneering work of Lotka and Volterra $[25,27,30,36]$. A huge number of prey-predator models are proposed and several interesting dynamical features are investigated. Different modeling approaches are adopted to analyze the interaction between prey and predator populations. Depending upon the nature of interaction and parameter values, some investigation reported sustained chaotic oscillation in all components of the model under consideration. The observed chaotic dynamics are supported by laboratory experiments as well as field data. On the other hand, research in the area of mathematical epidemiology have received considerable attention after the pioneering work of Kermack-McKendric on SIRS model, where S, I, R stands for susceptible, infective and recovered population respectively [23]. Epidemic models are investigated thoroughly by

*Corresponding author. E-mail: malayb@iitk.ac.in

(C) EDP Sciences, 2012 
several researchers to understand the nature and progression of diseases and also to suggest the suitable control mechanism by vaccination or any other means. Combining these two fields, researchers engaged themselves with the study of eco-epidemiological models during last two decades $[4,7,13,20,21,29,34,35]$. Ecological models are extended to eco-epidemiological models by dividing one or more species into susceptible and infective class. So far as our knowledge goes, most of the eco-epidemic models are dealt with susceptible and infective classes only, SIR, SIER types of classification are not adopted till to date.

Eco-epidemic models with prey-predator type interaction mainly dealt with the role of predation on susceptible and infected prey population to slow down or eradication of disease. As the functional response is the sole link between prey and predator population, the choice of functional response to model the interaction between susceptible prey and predators as well as infective prey and predators. In some situations the infective preys are more vulnerable or easily accessible to the predators. On the other hand, the transmission of disease in to the predators due to predation of infected prey and reduced predation ability of infected predators are also important issues to understand the dynamics of concerned model. Although the division of species into subclasses increase the degree of complexity of the concerned interacting model but chaotic dynamics is reported in few cases [32].

In 2001, Chattopadhyay and Bairagi [11] proposed and analyzed an eco-epidemic model to study the interaction between Tilapia fish population and Pelicans at Salton Sea. They have reported that the Tilapia fishes are infected through Avian Botulism bacteria and hence results in huge death of the fish species. A detailed discussion of the habitat, nature and mechanism of infection, disease related death of Tilapia fishes and massive death of Pelicans due to predation of infected fishes can be found in [11]. The authors considered a three dimensional nonlinear coupled ordinary differential equation model to study the interaction between susceptible Tilapia population, infected Tilapia population and Pelican population where Pelicans are considered as predator of infected fish population only. The model was built up with the assumption that infected Tilapia fishes come to surface of the sea and becomes more vulnerable for predation by the Pelicans. For the concerned model, authors have obtained the conditions for local asymptotic stability of all equilibrium points and global stability of axial equilibrium point only and derived the condition for persistence of all species. Considering search rate of predators as key parameter, it was reported that the co-existing steady-state losses its stability with the increase of search rate level and oscillatory dynamics sets in. It was suggested that proper harvesting of infected Tilapia fishes should be implemented to save the Pelicans.

Recently, Upadhyay et al [33] revisited the model proposed in [11] and observed the chaotic dynamics for the same model under certain choice of parameter values. To obtain the chaotic oscillation for susceptible and infected Tilapia fishes and Pelicans, authors have considered growth rate of Tilapia fishes, their environmental carrying capacity and half-saturation constant as bifurcation parameters. They have reported that the variation of other parameters is not responsible for chaotic oscillation. It was reported that the chaotic dynamics is quite relevant as the nutrient concentration of the sea water increases during late summer and which in turn increases the carrying capacity and growth rate of Tilapia fish population. This increased growth of fish population drives the system towards the chaotic regime. Apart from these observations, authors have reported that chaotic behavior is mixed with stable periodic coexistence. This result is not true and we are intended to nullify this possibility by showing the period doubling route to chaos, which was overlooked in [33]. We also derive the condition required for Hopf-bifurcation which is responsible for the initiation of small amplitude periodic solution around co-existing steady-state.

Dynamical analysis of eco-epidemiological models within deterministic setup have received attention from researchers but the fluctuation of environment and/or demographic variation in the species involved with the model remains an unexplored area apart from few investigations. On the other hand, various stochastic modeling approach is adopted for mathematical models of epidemiology $[3,6,8-10,12,18]$. There are three different modeling approaches to study the environmental and demographic variability to understand the spread and progress of epidemic diseases. In the first modeling approach, a discrete state space model is built up based upon the different possible changes in the components involved with the system under consideration within very small time interval. In the second modeling approach, a 
discrete-time stochastic model is constructed first based on the observed independent random changes involved with the system and then approximated by a system of stochastic differential equation. The third approach is the most well-known procedure adopted for the formulation of stochastic differential equation models. In this approach, specific functional forms are chosen for drift and diffusion matrix for the dynamical system under consideration [5]. Due to limitations of mathematical machineries to analyze these stochastic models, most of the investigations in this direction are based upon exhaustive numerical simulations and interpretation of the results obtained through numerical simulations for suitable range of parameter values.

The main objective of the present paper is two fold, firstly we are interested to study the global dynamics of the deterministic model proposed in [11] and establish the period doubling route to chaos. Secondly, we construct the stochastic analogue of the existing deterministic model with the help of newly proposed stochastic modeling approach by Allen et al to understand the effect demographic fluctuation in fish species as well as in Pelican birds to shape the resulting dynamics. The main objective of the present paper is two fold, firstly we are interested to study the global dynamics of the deterministic model proposed in [11] and establish the period doubling route to chaos. Apart from the investigation for route to chaos, we also perform local stability and Hopf-bifurcation analysis with the contact rate and death rate of predators as bifurcation parameter. Secondly, we construct the stochastic analogue of the existing deterministic model with the help of newly proposed stochastic modeling approach by Allen et al. to understand the effect demographic fluctuation in fish species as well as in Pelican birds to shape the resulting dynamics. In the next section, we recall basic results for the deterministic model and obtain the criteria for Hopf-bifurcation and then establish the period doubling route to chaos. We describe the construction of stochastic model in section 3. Results obtained through exhaustive numerical simulations of the stochastic model are given in section 4. In concluding section we discuss the basic outcomes of present analysis and future direction of work.

\section{Basic Model}

In this section, we consider the same three dimensional model as proposed by Chattopadhyay and Bairagi [11] and further investigated by Upadhyay et al [33]. Deterministic model consists of mainly two species, Tilapia fishes and Pelican birds. Fish population is divided into two classes, susceptible and infected where susceptible fishes are getting infection in contact with infected individuals and disease is transmitted by following the law of mass action. In [11], authors have assumed that the susceptible Tilapia fishes are only capable to give birth of new individuals but infected fishes compete with susceptible fishes for the food. It was further assumed that the Pelicans are catching infected Tilapia fishes only as they come to the surface of sea after getting infected and consumption of infected fishes by Pelicans follow Holling type-II functional response. $s(t), i(t)$ and $p(t)$ denote susceptible fish population, infected fish population and Pelican population respectively at any instant of time ' $t$ '. The dynamic interaction is governed by the following three dimensional coupled nonlinear ordinary differential equations,

$$
\begin{aligned}
& \frac{d s}{d t}=r s\left(1-\frac{s+i}{k}\right)-\lambda s i, \\
& \frac{d i}{d t}=\lambda s i-\mu i-\frac{m i p}{i+a}, \\
& \frac{d p}{d t}=\frac{\theta i p}{i+a}-\delta p,
\end{aligned}
$$

subjected to positive initial conditions $s(0)=s_{0}>0, i(0)=i_{0}>0$ and $p(0)=p_{0}>0$. All parameters involved with the model are positive constants and their ecological interpretation are in order, $r$ is the intrinsic growth rate of susceptible fishes, $k$ denotes the environmental carrying capacity for total fish population, $\lambda$ is the contact rate, $\mu$ is intrinsic death rate of infected Tilapia fishes, $m$ is the rate of 
predation, $a$ denotes half-saturation constant, $\theta$ is the growth rate of Pelicans due to predation and $\delta$ is the death rate of Pelicans. Here the parameter $\delta$ includes natural death rate and death rate due to predation of infected Tilapia fishes. Existence-uniqueness and boundedness of solutions for the model (2.1) - (2.3) are discussed in [11].

$E_{0}(0,0,0)$ is trivial equilibrium point, $E_{1}(k, 0,0)$ is the disease free equilibrium point, predator free equilibrium point is $E_{2}\left(\frac{\mu}{\lambda}, \frac{r(\lambda k-\mu)}{\lambda(\mu+\lambda k)}, 0\right)$ and $E_{*}\left(s_{*}, i_{*}, p_{*}\right)$ is the interior equilibrium point whose components are given by

$$
s_{*}=k-\frac{a \delta(r+\lambda k)}{r(\theta-\delta)}, i_{*}=\frac{a \delta}{\theta-\delta}, p_{*}=\frac{1}{m}\left(a+i_{*}\right)\left(\lambda s_{*}-\mu\right) .
$$

$E_{0}$ and $E_{1}$ exist without any parametric restriction, feasible existence of $E_{2}$ demands the restriction $\lambda>\mu / k$ and satisfaction of parametric restrictions $\lambda k>\mu$ and $\theta>\delta+\frac{\lambda a \delta(r+\lambda k)}{r(\lambda k-\mu)}$ ensure the existence of interior equilibrium point. Local asymptotic stability results of all equilibria were investigated by Chattopadhyay and Bairagi [11]. Here we discuss the local stability of $E_{*}$ only as it is required for the Hopf-bifurcation analysis. Further we like to remark that the stability conditions for $E_{*}$ derived in [33] are sufficient but not necessary. Those conditions are rather stronger conditions compared to the conditions required.

The Jacobian matrix for the model $(2.1)$ - (2.3) evaluated at $E_{*}$ is given by

$$
J_{*}=\left[\begin{array}{ccc}
-\frac{r}{k} s_{*} & -\left(\frac{r}{k}+\lambda\right) s_{*} & 0 \\
\frac{\lambda a \delta}{\theta-\delta} & \frac{\delta}{\theta}\left(\lambda s_{*}-\mu\right) & -\frac{m \delta}{\theta} \\
0 & \frac{\theta-\delta}{m}\left(\lambda s_{*}-\mu\right) & 0
\end{array}\right],
$$

and the characteristic equation associated with $J_{*}$ is given by

$$
\Lambda^{3}+A_{1} \Lambda^{2}+A_{2} \Lambda+A_{3}=0
$$

where

$$
\begin{gathered}
A_{1}=\frac{r}{k} s_{*}+\frac{\mu \delta}{\theta}-\frac{\lambda \delta}{\theta} s_{*}, \\
A_{2}=\frac{\delta}{\theta}(\theta-\delta)\left(\lambda s_{*}-\mu\right)+\left(\frac{r}{k}+\lambda\right) \frac{\lambda a \delta}{\theta-\delta} s_{*}-\frac{r \delta}{k \theta}\left(\lambda s_{*}-\mu\right) s_{*}, \\
A_{3}=\frac{r \delta(\theta-\delta)}{k \theta}\left(\lambda s_{*}-\mu\right) s_{*}>0 .
\end{gathered}
$$

According to the Routh-Hurwitz criteria, $E_{*}$ is locally asymptotically stable if $A_{1}>0$ and $A_{1} A_{2}>A_{3}$. It is quite difficult to find explicit parametric restriction for local asymptotic stability of $E_{*}$ but we can discuss this issue for some specific choice of system parameters. For this purpose, we choose the parameter values $k=400, \lambda=.06, \mu=3.4, m=15.5, a=15, \theta=10, \delta=8.3$ and consider $r$ as bifurcation parameter. Units of the parameters, suitability of their magnitudes, and ecological justifications are discussed exhaustively in [11,33]. For this choice, interior equilibrium point is $E_{*}\left(\frac{326.76 r-1757.65}{r}, 73.24, \frac{92.25 r-600.33}{r}\right)$ and feasibility condition requires $r>6.5$. $A_{1}=.82 r-17.84+\frac{87.53}{r}$ and it is positive for $r \in(7.5,7.44) \cup(14.41, \infty)$ and $A_{3}$ is positive whenever $E_{*}$ is ecologically feasible. So for local asymptotic stability of $E_{*}$, we have to check the sign of $A_{1} A_{2}-A_{3}$. Evaluating $A_{1} A_{2}-A_{3}$ as a function of ' $r$ ' only, we get

$$
A_{1} A_{2}-A_{3}=-6.04 r^{2}+293.33 r-5171.47+\frac{36419.09}{r}-\frac{87252.62}{r^{2}},
$$

which is positive for $r \in(5.55,7.28)$. For $r>7.28$ the quantity $A_{1} A_{2}-A_{3}$ remains negative. At $r=r_{*}=7.277970290$ we have $A_{1}>0, A_{3}>0$ and $A_{1} A_{2}-A_{3}=0$ with $\left.\frac{d}{d r}\left(A_{1} A_{2}-A_{3}\right)\right|_{r=r_{*}} \neq 0$. 
Hence at $r=r_{*}$, all conditions for Hopf-bifurcation are satisfied and the interior equilibrium point looses its stability $[26,28]$. As a result small amplitude periodic solution bifurcates from $E_{*}$. Similar type analysis can be carried out for choosing other parameter as bifurcation parameter.
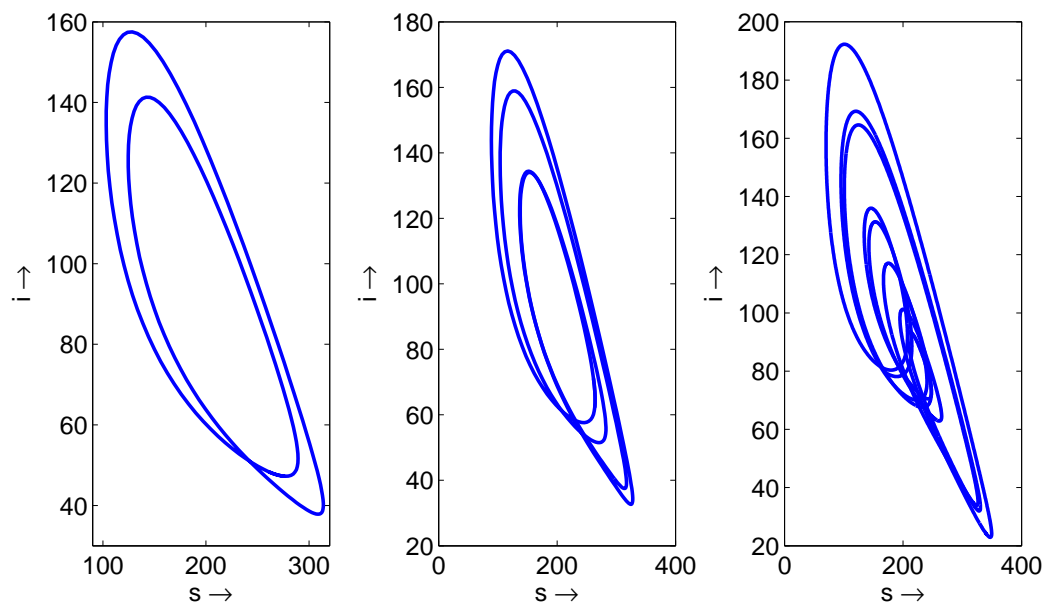

Figure 1. Projection of periodic attractors on $s i$-plane for different values of $r$, showing period doubling of the limit-cycle. Periodic solutions are depicted with period 2 (for $r=20.57$ ), period 4 (for $r=21.2543$ ) and period 8 (for $r=21.31$ ).

For chosen set of parameter values, authors [33] have reported that $E_{*}$ is stable for $r \in[5,7.25]$, stable limit cycle exists for $r \in[7.30,21.10]$, chaotic attractor exists for $r \in[21.15,22.20]$, stable limit cycle exists for $r \in[22.25,23.35]$, chaotic dynamics observe for $r \in[23.39,23.86]$, stable limit cycle exists for $r \in[23.87,23.97]$ and again chaotic dynamics observed for $r \in[23.98,25.00]$. This result is not true as feasibility of interior equilibrium point demands $r>6.5$ and limit-cycle arises through Hopf-bifurcation at $r \equiv r_{*}=7.277970290$. Further, their claim for alternative occurrence of stable limit cycle and chaotic attractor is also not true. To establish our claim, now we show the period doubling route to chaos with the variation of $r$.

We have performed numerical simulations for the model $(2.1)-(2.3)$ with $\Delta t=.0001$ and used explicit 4 -th order Runge-Kutta scheme and for initial condition $\left(s_{0}, i_{0}, p_{0}\right)=(100,80,20)$. The interior equilibrium point $E_{*}$ is locally asymptotically stable for $6.5<r<7.277970290$. Thorough numerical investigation reveals that the oscillatory solution with period one arising through Hopf-bifurcation at $r=7.277970290$ and sustain up to $r<20.57$. First period doubling occurs at $r=20.57230178$ and we observe periodic solution with period-2 whenever $r$ belongs to $(20.57230178,21.2541287)$. Next period doubling occurs at $r=21.2541287$ and we find periodic solution with period-4 for $r \in(21.2541287,21.3127834)$. Further increment in the magnitude of $r$ results in chaotic oscillation. Period-doubling of limit cycle with varying $r$ is presented in Fig. 1. The bifurcation diagram is drawn for $s$-component against the bifurcation parameter and is presented in Fig. 2. The chaotic attractor obtained for $r=24$ in three dimensional space is shown in Fig. 3 .

\subsection{Two Hopf-bifurcation with $\lambda$ as parameter}

In this subsection, we consider the contact rate as bifurcation parameter. For the bifurcation analysis, we choose other parameter values as $r=7, k=400, \mu=3.4, m=15.5, a=15, \theta=10$ and $\delta=8.3$. With $\lambda$ as parameter, interior equilibrium point is given by $E_{*}(326.76-4184.87 \lambda, 73.24,1860.14 \lambda-$ $\left.23822.81 \lambda^{2}-19.35\right)$ and feasibility of coexisting steady-state determines the admissible range for $\lambda$ as 


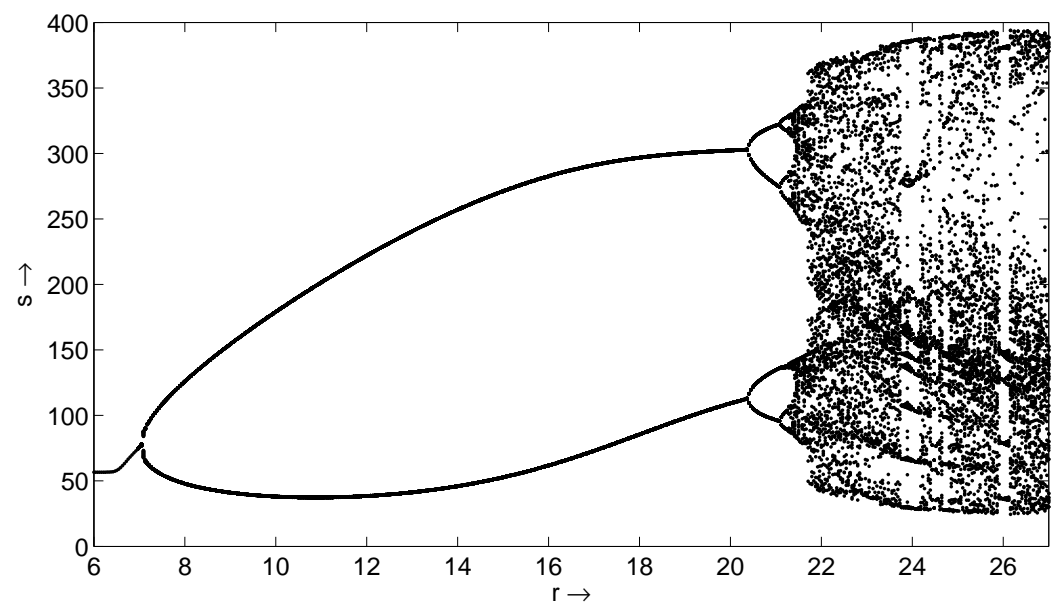

FIGURE 2. Bifurcation diagram for susceptible fish species for a range of values of $r$.

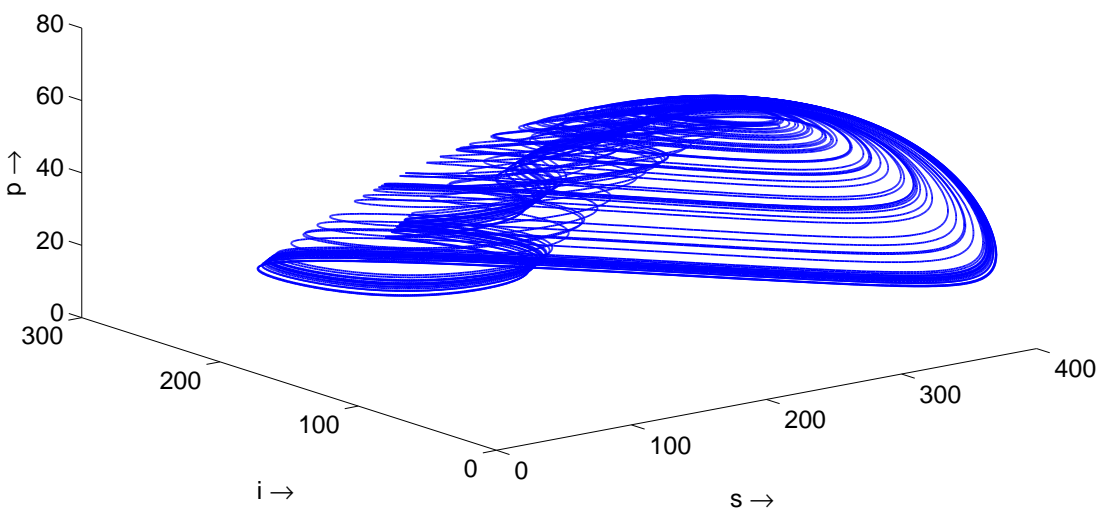

FiguRE 3. Chaotic attractor in three dimensional space obtained for $r=24$ and other parameter values as mentioned in the text.

$I_{\lambda} \equiv(.01236228277, .06572004655)$. Now we check the local stability properties of $E_{*}$ for $\lambda \in I_{\lambda}$. We mentioned earlier that $A_{3}>0$ whenever $E_{*}$ is biologically feasible, so here we calculate only $A_{1}$ and $\Delta=A_{1} A_{2}-A_{3}$ in terms of $\lambda$ to understand the stability behavior. Substituting the components of $E_{*}$ in (2.6) and (2.8), we get after some algebraic simplification,

$$
\begin{aligned}
A_{1} & =3473.45 \lambda^{2}-344.45 \lambda+8.54, \\
\Delta & =-1948114034 \lambda^{5}+375152821.8 \lambda^{4}-26315980.57 \lambda^{3}+856662.56 \lambda^{2}-14390.03 \lambda+124.28 .
\end{aligned}
$$

$A_{1}>0$ for all $\lambda \in I_{\lambda}$ and $\Delta$ has two zeros in $I_{\lambda}$ (see Fig. 4). $\lambda \equiv \lambda_{H 1}=.03673203067$ and $\lambda \equiv \lambda_{H 2}=.05601411665$ are two zeros of $\Delta$ and system under goes Hopf-bifurcation at $\lambda=\lambda_{H 1}$ and $\lambda=\lambda_{H 2}$. Interior equilibrium losses stability at $\lambda_{H 1}$ and regains at $\lambda_{H 2}$. Within the admissible range of $\lambda, E_{*}$ is locally asymptotically stable for $\lambda \in\left(.01236228277, \lambda_{H 1}\right)$ and $\lambda \in\left(\lambda_{H 2}, .06572004655\right)$. Interior equilibrium is unstable for $\lambda \in\left(\lambda_{H 1}, \lambda_{H 2}\right)$ and we find oscillatory coexistence all species, all 
other boundary equilibria are unstable. One can easily verify the transevrsality condition, here we omit the verification from the sake of brevity. Bifurcation diagram for three species against $\lambda$ is presented in Fig. 5. Bifurcation diagram clearly shows the change in equilibrium population level with increasing values of $\lambda$.

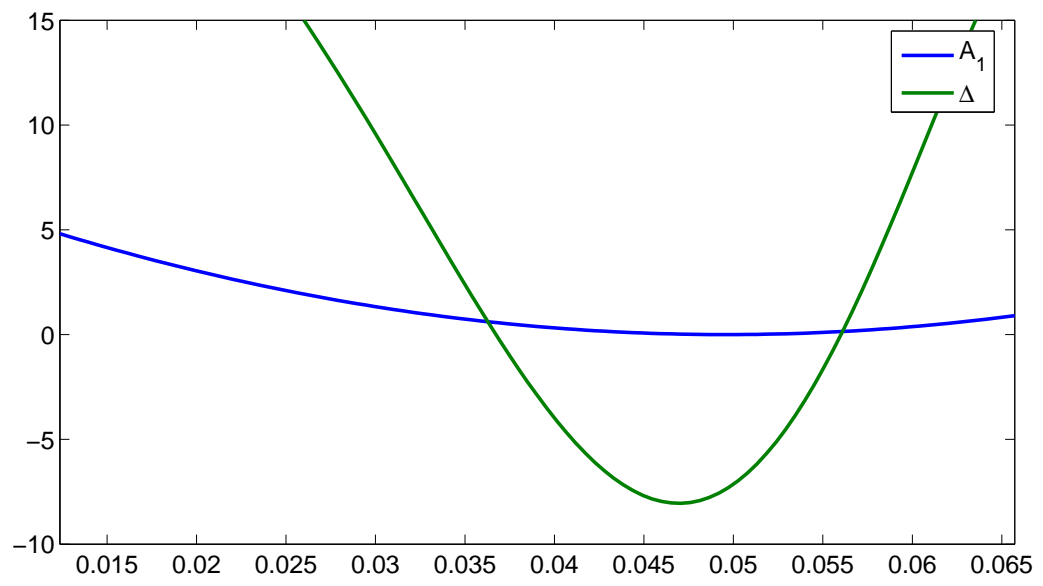

Figure 4. Plot of $A_{1}$ and $\Delta=A_{1} A_{2}-A_{3}$ against lambda for $\lambda \in$ $(.01236228277, .06572004655)$.
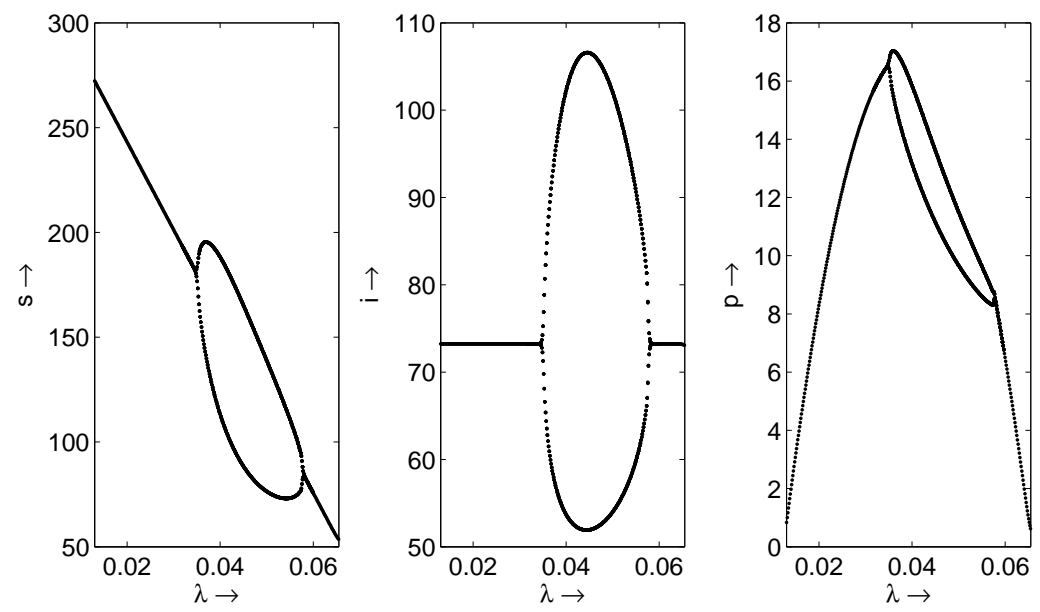

FiguRE 5. Bifurcation diagram for three species is obtained against $\lambda$ varying in the interval $I_{\lambda}$. Interior equilibrium bifurcates into periodic solution at $\lambda_{H 1}$ and restored at $\lambda_{H 2}$. 


\subsection{Backward Hopf-bifurcation}

Considering the intrinsic growth rate of susceptible fish population and contact rate as bifurcation parameters, we have observed that the interior equilibrium point losses its stability through Hopf-bifurcation with increasing values of them. In case of $\lambda$ as bifurcation parameter, periodic oscillation disappears through second Hopf-bifurcation. In this subsection, we consider the death rate of Pelicans, that is, $\delta$ as parameter and keeping other parameters fixed at $r=7, k=400, \lambda=.06, m=15.5, a=15, \mu=3.4$ and $\theta=10$. Feasible existence of interior equilibrium point $E_{*}\left(\frac{4000-466.43 \delta}{10-\delta}, \frac{15 \delta}{10-\delta}, \frac{1993.55-237.93 \delta}{(10-\delta)^{2}}\right)$ imposes the restriction $\delta<8.378849506$. Proceeding in a similar fashion as above, we calculate the following quantities in terms of $\delta$,

$$
\begin{aligned}
A_{1} & =\frac{2.46 \delta^{2}-28.76 \delta+70}{10-\delta}, \\
\Delta & =-\frac{6.04 \delta^{6}-172.85 \delta^{5}+1720.93 \delta^{4}-5501.8 \delta^{3}-12554.77 \delta^{2}+81410 \delta+.00048}{(10-\delta)^{3}} .
\end{aligned}
$$

$A_{3}$ is positive until $E_{*}$ is feasible. There exist only one value of $\delta$ within its feasible range at which $A_{1}>0$ and $\Delta=0$. This critical value is $\delta \equiv \delta_{H}=8.263939390$. $E_{*}$ is unstable for $\delta<\delta_{H}$ and it stabilizes through Hopf-bifurcation and coexisting steady-state is locally asymptotically stable for $\delta \in\left(\delta_{H}, 8.378849506\right)$. As $\Delta<0$ for all positive $\delta$ having magnitude less than $\delta_{H}$, there is no chance of further Hopf-bifurcation through which $E_{*}$ may regain its stability. The stabilization of interior equilibrium through Hopf-bifurcation as $\delta$ crosses the threshold magnitude $\delta_{H}$ from lower to higher is labeled here as backward Hopf-bifurcation. In most of the ecological models, interior equilibrium points become unstable through Hopf-bifurcation when the responsible parameter passes through the threshold magnitude from lower to higher. This is the reason for the Hopf-bifurcation occurring at $\delta=\delta_{H}$ is mentioned here as backward bifurcation. We like to mention here that the notion of 'backward bifurcation' used here is completely different compared to the same phrase used in literature for epidemic models [19]. Fig. 6 shows oscillatory co-existence of three species when $\delta<\delta_{H}$.
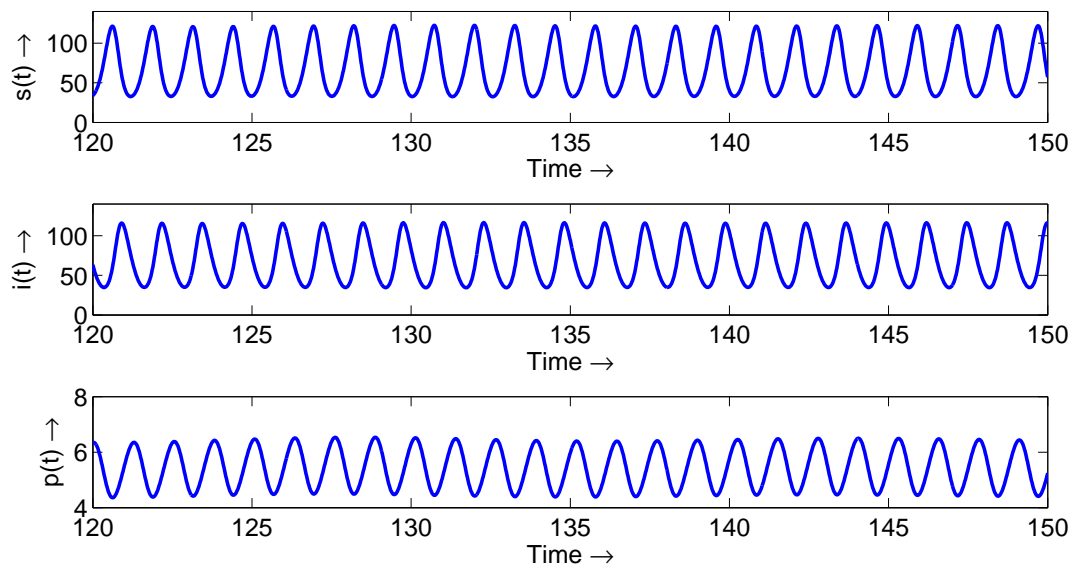

FIGURE 6. Hopf-bifurcating periodic solution obtained through numerical simulation with $\delta=8<\Delta_{H}$ and other parameter values as mentioned in text. 


\section{Stochastic Model Formulation}

In this section, we consider a stochastic model corresponding to the deterministic model considered in previous section. Among various possible approaches for constructing stochastic model, here we consider the demographic stochasticity for fish and bird populations. Here we derive the stochastic differential equation model directly from the deterministic model based upon the fact that all possible changes can be clearly identified separately.

Let $S(t), I(t)$ and $P(t)$ denote continuous time random variables corresponding to $s(t), i(t)$ and $p(t)$ respectively, such that $X(t)=(S(t), I(t), P(t))^{T}$ is a random vector defined on an appropriately defined sample space, where

$$
S(t), I(t), P(t) \in[0, \infty), \quad t \in[0, \infty) .
$$

Assume that the time interval $\Delta t$ is sufficiently small and at least one change can occur in the time interval $[t, t+\Delta t)$. The small change in random vector is denoted by $\Delta X(t) \equiv(\Delta S(t), \Delta I(t), \Delta P(t))^{T}$, where $\Delta X(t)=X(t+\Delta t)-X(t)$. The $j$-th possible change is denoted by $(\Delta X)_{j}$. The time interval $\Delta t$ is chosen sufficiently small such that possible change in any species is at most one unit. Keeping in mind the deterministic model, the possible changes of random vector are given in the following table, where terms of order $(\Delta t)^{2}$ and higher are neglected.

Table 1. Possible Changes in $\Delta X$

\begin{tabular}{c|c|c}
\hline$j$ & $(\Delta X)_{j}^{T}$ & Probability \\
\hline 1 & $(1,0,0)$ & $p_{1}=r S \Delta t=b_{1} \Delta t$ \\
2 & $(-1,0,0)$ & $p_{2}=\frac{r S(S+I)}{K} \Delta t=d_{1} \Delta t$ \\
3 & $(-1,1,0)$ & $p_{3}=\lambda I S \Delta t=m_{1} \Delta t$ \\
4 & $(0,-1,0)$ & $p_{4}=\left(\frac{m P I}{I+a}+\mu I\right) \Delta t=d_{2} \Delta t$ \\
5 & $(0,0,1)$ & $p_{5}=\frac{\theta I P}{I+a} \Delta t=b_{3} \Delta t$ \\
6 & $(0,0,-1)$ & $p_{6}=\delta P \Delta t=d_{3} \Delta t$ \\
7 & $(0,0,0)$ & $1-\sum_{j=0}^{6} p_{j}$ \\
\hline
\end{tabular}

Now we are in a position to compute the expectation and covariance of $\Delta X$ for sufficiently small $\Delta t$. The expectation of $\Delta X$ is

$$
\begin{gathered}
\mathbb{E}(\Delta X) \approx\left[\left[\begin{array}{l}
1 \\
0 \\
0
\end{array}\right] b_{1}+\left[\begin{array}{c}
-1 \\
0 \\
0
\end{array}\right] d_{1}+\left[\begin{array}{c}
-1 \\
1 \\
0
\end{array}\right] m_{1}+\left[\begin{array}{c}
0 \\
-1 \\
0
\end{array}\right] d_{2}+\left[\begin{array}{l}
0 \\
0 \\
1
\end{array}\right] b_{3}+\left[\begin{array}{c}
0 \\
0 \\
-1
\end{array}\right] d_{3}\right] \Delta t \\
=\left[\begin{array}{c}
b_{1}-d_{1}-m_{1} \\
m_{1}-d_{2} \\
b_{3}-d_{3}
\end{array}\right] \Delta t=\left[\begin{array}{c}
\mu_{1} \\
\mu_{2} \\
\mu_{3}
\end{array}\right] \Delta t \equiv \mu \Delta t,
\end{gathered}
$$

and covariance matrix for $\Delta X$ is given by,

$$
\Sigma \Delta t=E\left[\Delta X(\Delta X)^{T}\right]-E[\Delta X](E[\Delta X])^{T} \approx E\left[\Delta X(\Delta X)^{T}\right]=\sum_{j=1}^{6} p_{j}(\Delta X)_{j}\left((\Delta X)_{j}\right)^{T} .
$$

The term $E[\Delta X]\left(E[\Delta X]^{T}\right)$ is neglected as it is of order $(\Delta t)^{2}$. Using expressions for $(\Delta X)_{j}, 1 \leq j \leq 6$ we get,

$$
\Sigma \Delta t=\left(\begin{array}{ccc}
r S+\frac{r S(S+I)}{K}+\lambda I S & -\lambda I S & 0 \\
-\lambda I S & \lambda I S+\frac{m P I}{I+a}+\mu I & 0 \\
0 & 0 & \frac{\theta I P}{I+a}+\delta P
\end{array}\right) \Delta t
$$


Further details regarding the calculations for mean and covariance of $\Delta X$ can be found in [2]. To formulate the stochastic model we need a square matrix ' $B$ ' such that $B^{2}=\Sigma$, but there is no unified way to do this for models involving three or more species. In [1,5], authors have discussed in detail how this difficulty can be tackled and provided an unified way to proceed further. Adapting the said approach discussed, we can define a matrix $G$ as follows,

$$
G=\left(\begin{array}{ccccc}
\sqrt{r S}-\sqrt{\frac{r}{K} S(S+I)} & -\sqrt{\lambda S I} & 0 & 0 & 0 \\
0 & 0 & \sqrt{\lambda S I} & -\sqrt{\frac{m P I}{I+a}+\mu I} & 0 \\
0 & 0 & 0 & 0 & \sqrt{\frac{\theta I P}{I+a}}-\sqrt{\delta P}
\end{array}\right),
$$

such that the condition $G G^{T}=\Sigma$ is satisfied. When $X(t)$ is sufficiently large compared to $\Delta t$, with out any loss of generality we can assume that the distribution of $\Delta X$ is normal with mean $\mu \Delta t$ and variance $\Sigma \Delta t$. In mathematical notations, we can write $\Delta X \sim N(\mu \Delta t, \Sigma \Delta t)[2,31]$. Let $\eta=\left(\eta_{1}, \eta_{2}, \cdots, \eta_{6}\right)$, where $\eta_{r} \sim N(0,1), 1 \leq r \leq 6$, denotes a six dimensional standard Wiener process $[14,15]$. Then $\mu \Delta t+G \eta \sqrt{\Delta t} \sim N\left(\mu \Delta t, G G^{T} \Delta t\right)$ and hence we can write,

$$
X(t+\Delta t)=X(t)+\Delta X=X(t)+\mu \Delta t+G \eta \sqrt{\Delta t} .
$$

$\eta_{r} \sqrt{\Delta t} \rightarrow d W_{r}$ as $\Delta t \rightarrow 0$ where $W_{r}$ denotes a Wiener process. The system mentioned in (3.1) converges to the following stochastic differential system in mean square sense as $\Delta t \rightarrow 0$,

$$
d X=\mu d t+G d W
$$

where $W=\left[W_{1}, W_{2}, \cdots, W_{6}\right]^{T}$ is a 6 -dimensional Wiener process where each components are independent to each other. Solution of (3.2) subjected to the positive initial condition $X(0)=[S(0), I(0), P(0)]^{T}$, (with $S(0), I(0), P(0)>0$ ) is an Itô process.

The stochastic differential equation model for susceptible Tilapia, infected Tilapia and Pelican is given by

$$
\begin{aligned}
d S & =\left[r S\left(1-\frac{S+I}{k}\right)-\lambda S I\right] d t+\sqrt{r S} d W_{1}-\sqrt{\frac{r}{K} S(S+I)} d W_{2}-\sqrt{\lambda S I} d W_{3}, \\
d I & =\left[\lambda S I-\mu I-\frac{m I P}{I+a}\right] d t+\sqrt{\lambda S I} d W_{3}-\sqrt{\frac{m P I}{I+a}+\mu I} d W_{4}, \\
d P & =\left[\frac{\theta I P}{I+a}-\delta P\right] d t+\sqrt{\frac{\theta I P}{I+a}} d W_{5}-\sqrt{\delta P} d W_{6},
\end{aligned}
$$

subjected to the positive initial condition $X(0)$.

\section{Numerical simulation results}

Apart from the existence and uniqueness of solution for the stochastic differential equations (3.3) - (3.5), we don't have any mathematical machinery to investigate the system analytically. The only possible way out is to solve the system numerically for chosen set of parameter values and we have to draw the conclusions based upon the obtained results. We solve the system (3.3) - (3.5) using Euler-Maruyama method with time stepping $\Delta t=10^{-6}$. Solutions obtained through Euler-Maruyama method has strong order of convergence $\gamma=\frac{1}{2}[22,24]$. One can verify the results with Milstein's method which has strong order of convergence $\gamma=1$, but the basic features will remain unaltered.

In section 2, we have discussed bifurcation behavior with respect to three parameters separately. For numerical simulations, we are interested to see the change in dynamic behavior for variation in $r$, $\lambda$ and $\delta$ one by one, keeping other parameters fixed at $k=400, \mu=3.4, m=15.5, a=15$ and 
$\theta=10$. First we check the dynamics of stochastic model for changing magnitude of $r$, we choose $\lambda=.06$ and $\delta=8.3$. For $r=7$, we have observed the coexisting steady-state for deterministic model is locally asymptotically stable. Solving the stochastic model (3.3) - (3.5) numerically with help of the said numerical scheme and chosen parameter values we find small amplitude fluctuation in all population, one simulation result is presented in Fig. 7. Two thousand sample paths are generated corresponding to the chosen set of parameter values and distribution of population at $t=100$ is presented in Fig. 8 . This distribution remains invariant at all future time and hence this distribution can be considered as stationary distribution. At this situation no species goes to extinction.

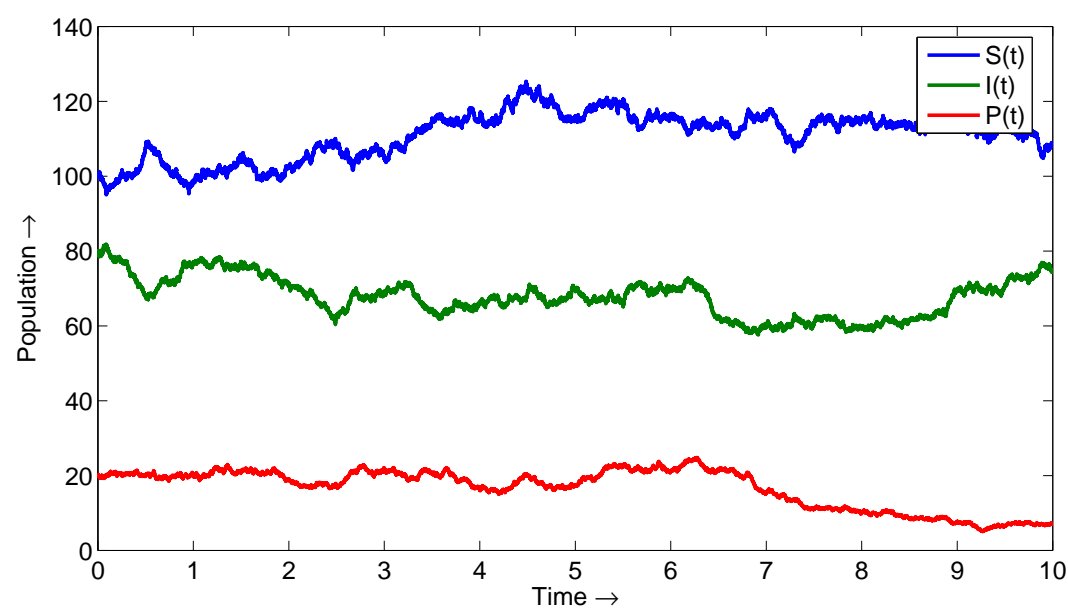

FiguRE 7. One sample path of the stochastic model plotted as a function of time for parameter values as mentioned in text and starting at $S(0)=100, I(0)=80, P(0)=20$.

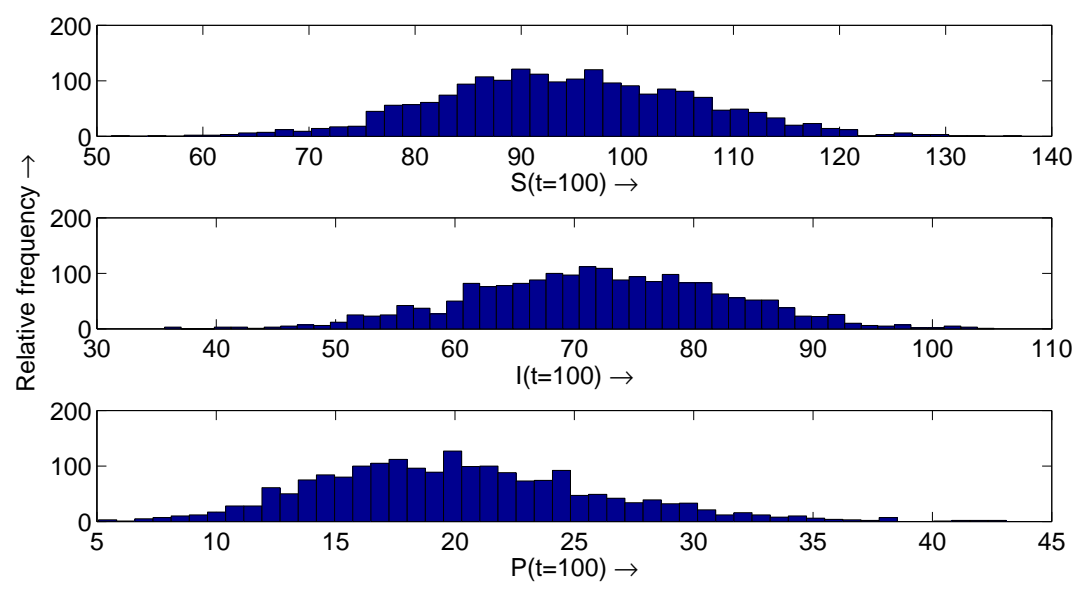

Figure 8 . The probability distribution of $S(t), I(t)$ and $P(t)$ at $t=100$ is presented. 
Within deterministic setup, we have observed the oscillatory coexistence for all species at $r=$ 7.27797029 when other parameters are fixed at the level mentioned in the last paragraph and all species coexist at oscillatory mode for values of $r$ higher than the threshold. For stochastic setup, non-equilibrium fluctuation is observed for values of $r$ greater than 7.1 (approximately). Rapidity and amplitude of fluctuation increases gradually with the increment of growth rate for the susceptible Tilapia fishes. Large amplitude fluctuation in fish population obtained from one numerical simulation run is presented in Fig. 9 when $r=8$. Repetition of same numerical simulation always produce similar type of fluctuation and Pelican species persists at very low density. Fluctuation in susceptible and infected fish populations are higher compared to Pelicans and the population density of Pelicans remains at low levels compared to other fish population. As a result the extinction scenario of Pelicans is observed. This extinction scenario arises for values of $r$ greater than 10. For $r=10$, we have calculated the average extinction time is equal to 8days (approx.) for Pelicans and average extinction time decreases with the increasing magnitude of $r$. Numerical simulation is carried out for higher values of $r$ and extinction of susceptible or infected fish population is rare. Extinction scenario of Pelican population obtained through one simulation run is presented in Fig. 10. It is worthy to mention here that no stationary distribution exist for any population as large amplitude fluctuation persists at all future time.

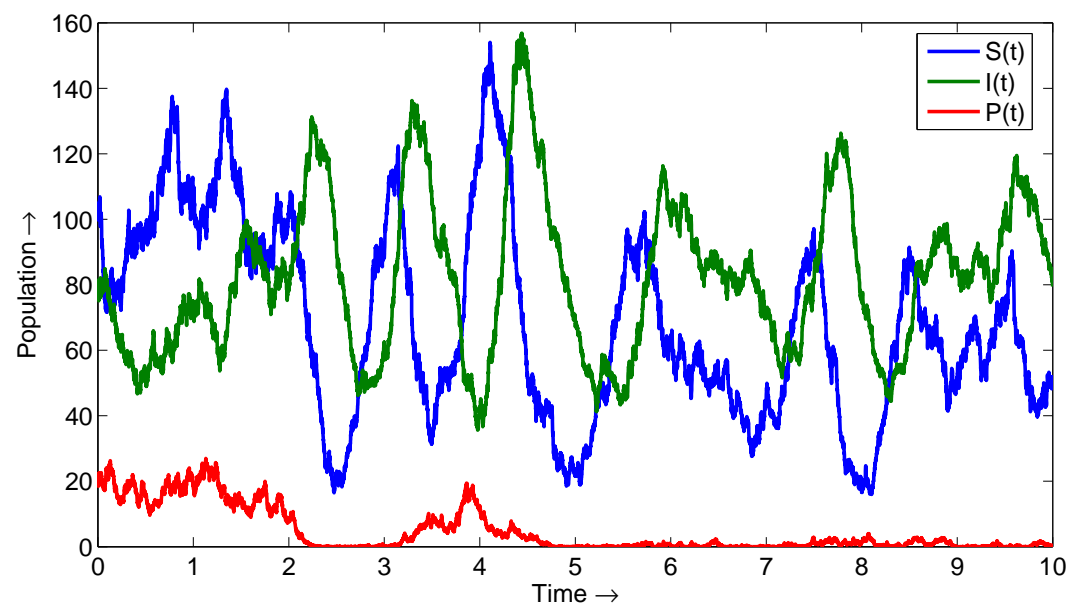

Figure 9. Fluctuation in susceptible and infected fish population obtained from numerical simulation of the stochastic differential system when $r=8$. Stochastic fluctuation in Pelicans population is low compared with other two populations.

In the subsection 2.1, we have observed that the equilibrium level for Pelicans changes significantly with the change in $\lambda$ whereas the equilibrium level of susceptible Tilapia fishes decreases with increasing $\lambda$ and that for infected Tilapia fishes remains constant. As the equilibrium level of Pelicans is very low for values of $\lambda$ near the end points of the interval $I_{\lambda}$, the persistence of all three species is observed when $\lambda$ takes values around the middle of $I_{\lambda}$. Chosen parameter values are $r=7, k=400, \mu=3.4, m=15.5$, $a=15, \theta=10$ and $\delta=8.3$. Coexistence of three species with high amplitude stochastic fluctuation is observed through numerical simulations and results of one simulation run is presented in Fig. 11. The numerical simulation is performed for $\lambda=.04$. This oscillatory coexistence persist for long time, although we are unable to find any stationary distribution due high amplitude fluctuation in all three components of the stochastic model.

Finally we consider the numerical simulation result for the stochastic model (3.3) - (3.5) with parameter values $r=7, k=400, \lambda=.06, \mu=3.4, m=15.5, a=15, \theta=10$ and $\delta=3$. Here the magnitude 


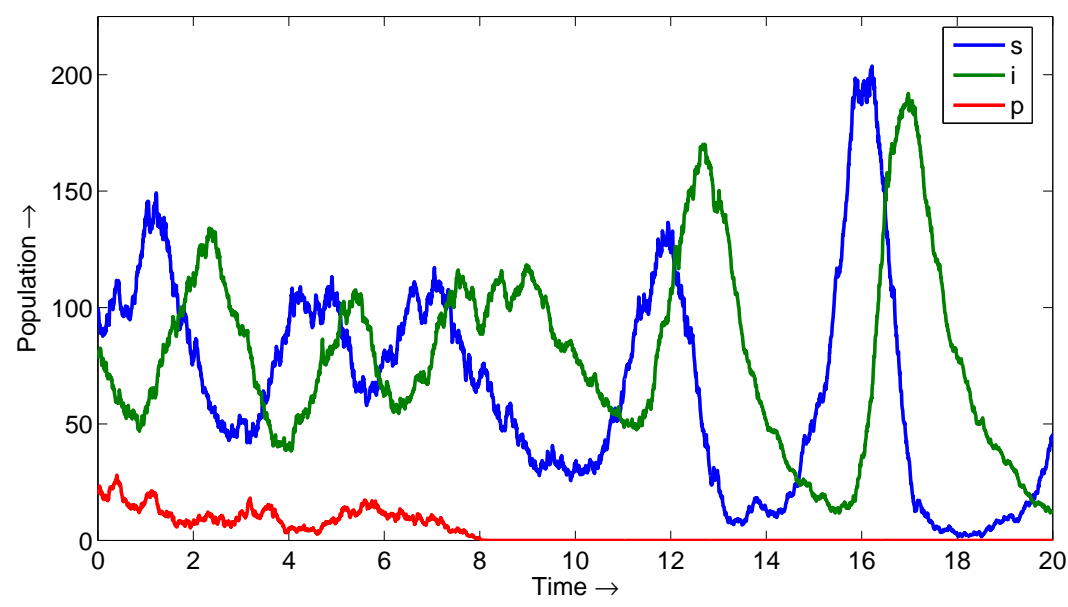

FiguRE 10. One simulation run shows extinction of $P$ at $t=8$ days approximately, other two populations exhibit large fluctuation. This simulation is performed for $r=10$ and other parameter values as mentioned in text.

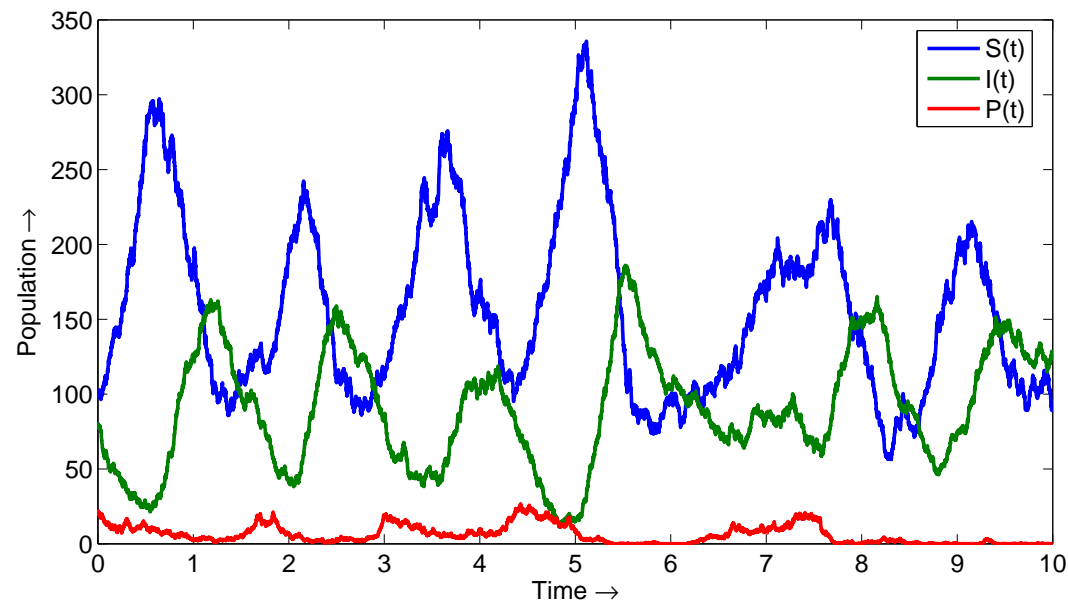

FIGURE 11. Stochastic high amplitude fluctuation is observed in susceptible fish, infected fish and Pelicans population, and three species persist at all future time.

of $\delta$ is quite less than the Hopf-bifurcation threshold $\delta_{H}$ for deterministic model. For the chosen set of parameter values, the interior equilibrium point for the model (2.1) - (2.3) is unstable but all three components coexist within stochastic setup. In this case also high amplitude stochastic fluctuation is observed for three species (see Fig. 12). Repeated simulation will produce similar dynamics and there is no chance extinction either for fish population or for Pelicans. Most interesting point is, the population of Pelicans some times higher than the infected fish population which is observed only for the parameter values mentioned at the beginning of this paragraph. Apart from this observation, the values attained by susceptible Tilapia fishes is also high compared the levels reported in Fig. 9 and Fig. 11. Mathematically speaking, the magnitude of equilibrium components of coexisting steady-state of deterministic model increases with decreasing magnitude of $\delta$. The mathematical expressions involved 
with the deterministic model are the same as the drift terms in stochastic model. As a result, the increasing magnitude of equilibrium level for deterministic model increases the amplitude of stochastic fluctuation. In this situation, the equilibrium level of Pelicans is significantly high for $\delta=3$ compared to earlier two sets of parameter values, where it was $\delta=8.3$. This higher equilibrium level decreases their chance of extinction. Another important observation is, the infected fish population maintain a low density for a longer period (see Fig. 12). As the death rate of Pelicans is low, the grazing pressure on infected fish population is quite high and as a result the increase in infected fish population requires a significant lowering of population density of their predators.

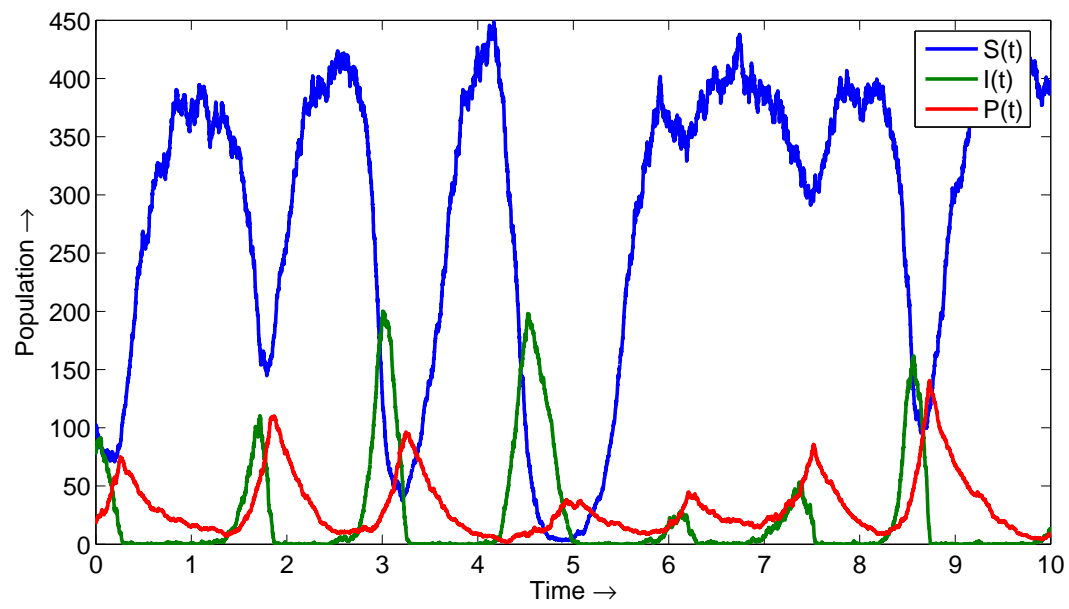

FigURE 12. Result of one numerical simulation run for the stochastic differential system (3.3) - (3.5) shows high amplitude non-equilibrium fluctuation in all three populations.

\subsection{Simulation with Gillespie algorithm}

Here we numerically simulate the sample paths for susceptible, infected fish species and Pelicans over the integer state space using Gillespie algorithm [16,17]. To apply this algorithm for the model under consideration we can recall that the change in population density for each species is due to either birth or death or infection. Events which are responsible to change the population density of three species are given in table - 1. To compare the simulation results obtained through Gillespie algorithm with the results obtained from Euler-Maruyama scheme for the stochastic system (3.3) - (3.5), we choose parameter values $r=7.27, k=400, \lambda=.06, \mu=3.4, m=15.5, a=15, \theta=10$ and $\delta=8.3$. In the Gillespie algorithm, the time to the next transition is a random variable which is distributed exponentially whose mean is the reciprocal of the sum of all transition rates. Outcome of one simulation run is presented in Fig. 13. Comparing this figure with Fig. 9 we see that fluctuations in susceptible and infected fish species are identical, but the population density of Pelicans stays at $P=1$ for some longer time intervals, see Fig. 14. A little increment in the magnitude of $r$ results to the more frequent extinction in Pelican species which was not observed during the numerical simulation runs of the stochastic system with EulerMaruyama scheme. This happens as for the chosen set of parameter values the population density of Pelican species always remain at lower level compared to susceptible and infected fish species. As a result $P(t)$ is mostly likely to take the value zero due to death when it was at the level one. But in case of numerical simulation of the stochastic model $(3.3)-(3.5)$, ' $P(t)$ ' can take any real values below ' 1 ' but greater than zero. This discrepancies can be avoided if we work with the realistic estimates of parameters and actual counts of individuals for each species rather than hypothetical set of parameter values and 
population density. Apart from this extinction scenario, the evolution of each species obtained through Euler-Maruyama scheme agrees with the Gillespie algorithm. Another important point we can mention here that the chosen time step $\Delta t=10^{-6}$ is approximately equal to the mean interevent time [2] of Gillespie algorithm. We have verified the results with other parameter sets and there are no significant difference between the simulation results obtained through Gillespie algorithm and Euler-Maruyama scheme. Only difference is that the probability of extinction in Pelican species is relatively high in case of the simulations performed with Gillespie algorithm. Figures of the simulations with other sets of parameter values with Gillespie algorithm are not provided here from the sake of brevity. Finally, we like to remark that the average interevent time obtained through Gillespie algorithm can be used as the suitable time step for numerical simulation of stochastic system over real state space.

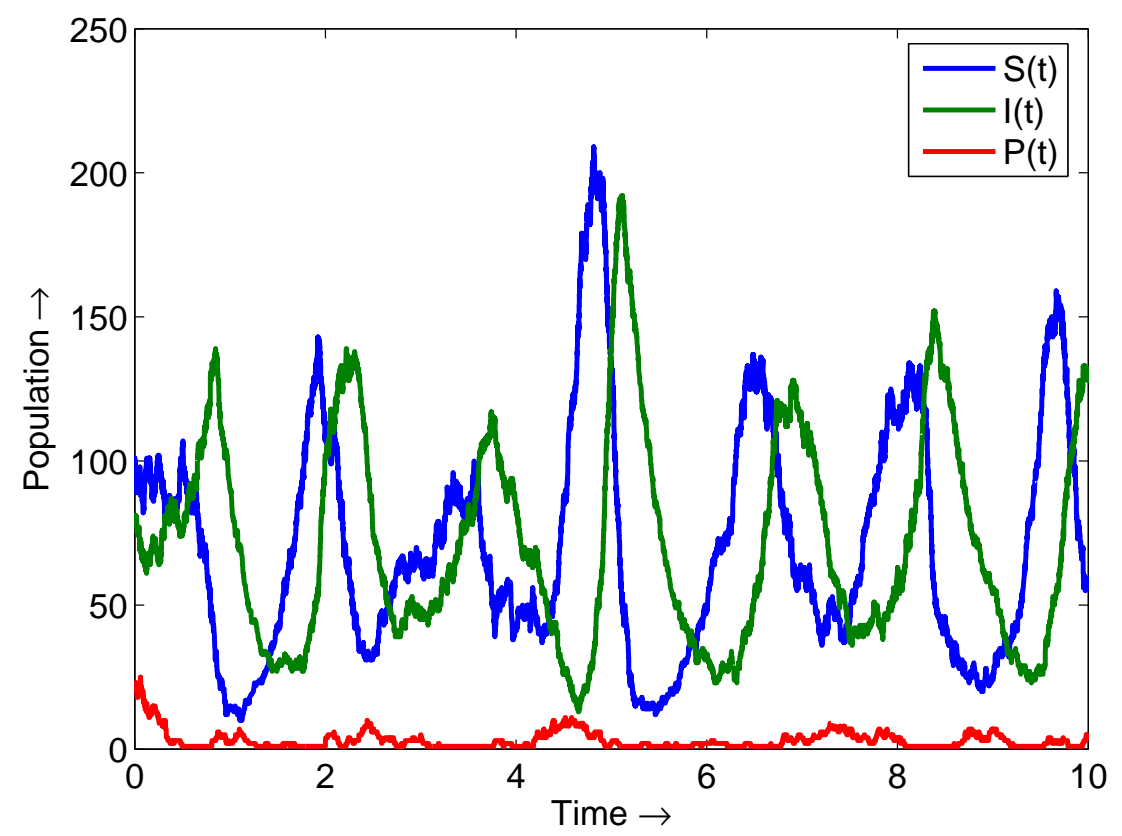

FIgURE 13. Time evolution of three species obtained from one simulation run by using Gillespie algorithm with the parameter values as mentioned in the text.

\section{Discussion}

In this paper, we have performed a rigorous bifurcation analysis for the deterministic model of dynamic interaction between Tilapia fish populations and Pelican birds in Salton Sea. The basic model was proposed by Chattopadhyay and Bairagi [11] and was further investigated in [33]. Basic model is an eco-epidemic model as the fish population is divided into susceptible and infected classes due to bacterial infection. As infected fishes are most vulnerable for predation and Pelicans are not clever enough to distinguish between infected and non-infected fish, model was constructed based upon the assumption that Pelicans predate only infected fishes. Upadhyay et al reported the chaotic dynamics for the same model but they were unable to detect the route to chaos. Here we have established the period doubling route to chaos when the growth rate of susceptible fishes is considered as bifurcation parameter. Interested readers can verify the chaotic oscillation observed for change in other parameter values (as discussed 


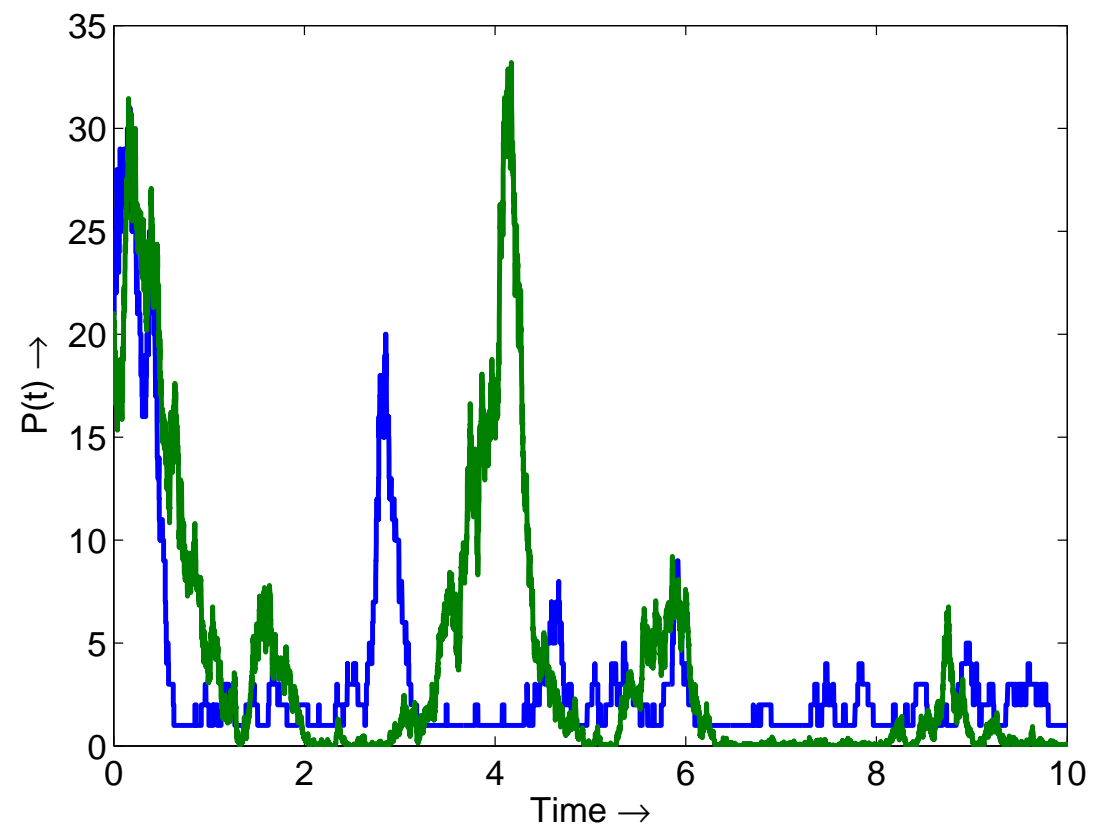

Figure 14. Time evolution of Pelican species obtained from one simulation runs using Euler-Maruyama scheme (green curve) and Gillespie algorithm (blue curve).

in [33]) and in all the cases chaotic dynamics appear through period-doubling and beyond the Hopfbifurcation threshold. We have also established that the repeated appearance of chaotic oscillation separated by stable periodic oscillation is not possible for the model under consideration and hence the dynamical behavior reported in [33] is not correct. The occurrence of two consecutive Hopf-bifurcations with increasing magnitude of contact rate is established here. Low death rate of Pelicans destabilizes the system but all three species coexist in oscillatory mode at all future time. When the magnitude of death rate for predators is greater than the Hopf-bifurcation threshold, we observe stable coexistence. Numerical examples are provided to validate the analytical findings.

Another important contribution of the present paper is the construction of stochastic differential system based upon the deterministic model setup. We have identified the different possible changes in population density that may take place within a small time interval and then constructed the stochastic differential system following the modeling approach proposed in [5]. The constructed stochastic model is capable to capture the demographic fluctuation in all three populations involved with the model. This modeling approach is valid when all system parameters are assumed to be invariant with the advancement of time. We have investigated the dynamics of stochastic model for various sets of parameter values with help of numerical simulations. So far as our knowledge goes, there are no available mathematical tools to study the dynamics of stochastic model constructed here analytically. To understand the dynamics of stochastic model, we have performed exhaustive numerical simulations. These simulations reveal some interesting features. First of all we have observed that Pelicans become extinct after 8days on average when growth rate of susceptible fish is greater than the Hopf-bifurcation threshold obtained from deterministic analysis, that is the coexisting steady-state for deterministic model is unstable. On the other hand, the coexistence of all species is observed for the values of contact rate, which correspond to the instability of deterministic steady-state. Similar dynamics is observed when death rate of Pelicans is varied. Thus the local asymptotic stability of deterministic model is not responsible for stochastic coexistence. Rather the magnitude of equilibrium levels obtained from the components of equilibrium 
point for deterministic model plays a crucial role. Further, the range of oscillation for three populations involved with the model we considered here also depends upon various rates of interactions. Finally, we like to remark that there is no one to one correspondence between stability of coexisting steady-state for deterministic model and stochastic persistence of the same model when constructed based upon the demographic stochasticity. Our analysis also detects the extinction of Pelicans within stochastic formalism, the local extinction of Pelicans was reported in [11], but not explored through any mathematical investigation. Further validation of the stochastic formalism needs estimation of parameters based upon the realistic data set.

Acknowledgements. The research work of first author is supported by the C. S. I. R., India. We are thankful to the anonymous reviewer for his/her constructive comments to improve the our work significantly.

\section{References}

[1] E. Allen. Modeling with Itô Stochastic Differential Equations. Springer, The Netherlands, 2007.

[2] L. J. S. Allen. An Introduction to Stochastic Processes with Applications to Biology. Pearson Eduction Inc., New Jercy, 2003.

[3] L. J. S. Allen, M. A. Jones, C. F. Martin. A discrete-time model with vaccination for a measles epidemic. Math. Biosci., 105 (1991), $111-131$.

[4] O. Arino, A. El. Abdllaoui, J. Mikram, J. Chattopadhyay. Infection on prey population may act as a biological control in ratio-dependent predator-prey model. Nonlinearity, 17 (2004), 1101 - 1116.

[5] E. J. Allen, L. J. S. Allen, A. Arciniega, P. Greenwood. Construction of equivalent stochastic differential equation models. Stoch. Anal. Appl., 26 (2008) $274-297$.

[6] F. G. Ball. Stochastic and deterministic models for SIS epidemics among a population partitioned into households. Math. Biosci., 156 ( 1999) $41-67$.

[7] E. Beltrami, T. O. Carroll. Modelling the role of viral disease in recurrent phytoplankton blooms. J. Math. Biol., 32 (1994) $857-863$.

[8] F. Brauer, C. Castillo-Chàvez. Mathematical Models in Population Biolgy and Epidemiology Springer-Verlag, New York, 2001.

[9] T. Britton. Stochastic epidemic models: A survey. Math. Biosci., 225 (2010) $24-35$.

[10] T. Britton, D. Lindenstrand. Epidemic modelling: Aspects where stochasticity matters. Math. Biosci., 222 (2009) 109 $-116$.

[11] J. Chattopadhyay, N. Bairagi. Pelicans at risk in Salton Sea - an eco-epidemiological model. Ecol. Model., 136 (2001) $103-112$.

[12] M. S. Chan, V. S. Isham. A stochastic model of schistosomiasis immuno-epidemiology. Math. Biosci., 151 (1998) 179 $-198$.

[13] H. I. Freedman. A model of predator-prey dynamics as modified by the action of parasite. Math. Biosci., 99 (1990) 143 -155 .

[14] T. C. Gard. Introduction to Stochastic Differential Equations. Marcel Decker, New York, 1987.

[15] C. W. Gardiner. Handbook of Stochastic Methods. Springer-Verlag, New York, 1983.

[16] D. T. Gillespie. A general method for numerically simulating the stochastic time evolution of coupled chemical reactions. J. Comp. Phy., 22 (1976) $403-434$.

[17] D. T. Gillespie. The chemical Langevin equation. J. Chem. Phy., 113 (2000) $297-306$.

[18] N. S. Goel, N. Richter-Dyn. Stochastic Models in Biology. Academic Press, New York, 1974.

[19] D. Greenhalgh, M. Griffiths. Backward bifurcation, equilibrium and stability phenomena in a three-stage extended BRSV epidemic model. J. Math. Biol., 59 (2009) 1 - 36.

[20] K. P. Hadeler, H. I. Freedman. Predator-prey population with parasitic infection. J. Math. Biol., 27 (1989) 609 - 631.

[21] M. Haque, D. Greenhalgh. A predator-prey model with disease in prey species only. M2AS, 30 (2006) $911-929$.

[22] D. J. Higham. An algorithmic introduction to numerical simulation of stochastic differential equations. SIAM Rev., $43(2001) 525-546$

[23] W. O. Kermack, A. G. McKendrick. A Contribution to the Mathematical Theory of Epidemics. Proc. Roy. Soc. Lond. A. 115 (1927) $700-721$.

[24] P. E. Kloeden, E. Platen. Numerical Solution of Stochastic Differential Equations. Springer, Berlin, 1999.

[25] M. Kot. Elements of Mathematical Biology. Cambridge University Press, Cambridge, 2001.

[26] Y. A. Kuznetsov. Elements of Applied Bifurcation Theory. Springer, Berlin, 1997.

[27] A. J. Lotka. Elements of physical biology. Williams \& Wilkins Co., Baltimore, 1925.

[28] J. Marsden, M. McCracken. The Hopf Bifurcation and its Applications. Springer, New York, 1976.

[29] H. Malchow, S. V. Petrovskii, E. Venturino. Spatiotemporal Patterns in Ecology and Epidemiology: Theory, Models and Simulations. Chapman \& Hall, London, 2008. 
[30] J. D. Murray. Mathematical Biology. Springer, New York, 1993.

[31] R. J. Serfling. Approximation Theorems of Mathematical Statistics. John Wiley \& Sons, New York, 1980.

[32] D. Stiefs, E. Venturino, U. Feudel. Evidence of chaos in eco-epidemic models. Math. Biosci. Eng., 6 (2009) 857 - 873.

[33] R. K. Upadhyay, N. Bairagi, K. Kundu, J. Chattopadhyay. Chaos in eco-epidemiological problem of the Salton Sea and its possible control. Appl. Math. Comput., 196 (2008) $392-401$.

[34] E. Venturino. The influence of diseases on Lotka-Volterra systems. Rocky Mountain Journal of Mathematics. 24 (1994) $381-402$.

[35] E. Venturino. Epidemics in predator-prey models: disease in the prey, In 'Mathematical Population Dynamics, Analysis of Heterogeneity'. 1, O. Arino, D. Axelrod, M. Kimmel, M. Langlais (Eds), Wnertz Publisher Ltd, Canada, 381 - 393 , 1995.

[36] V. Volterra. Variazioni e fluttuazioni del numero d'individui in specie animali conviventi. 2. Mem. R. Accad. Naz. dei Lincei. Ser. VI, 1926. 